\title{
Thirty-Day Readmission After Elective Colorectal Surgery for Colon Cancer: A Single-Center Cohort Study
}

\author{
Jun Seong Chung, Han Deok Kwak, Jae Kyun Ju \\ Department of Surgery, Chonnam National University Hospital, Gwangju, Korea
}

Purpose: There is a concern that enhanced recovery after surgery may affect other proposed quality measures, including the rate of readmission due to early discharge. We examine the 30-day readmission rate, risk factors associated with readmission after elective colorectal surgery for colon cancer, causes of readmission, disease-free survival (DFS), and overall survival (OS) in a single institution.

Methods: We retrospectively investigated 292 patients who underwent elective colorectal surgery for colon cancer between 2010 and 2015. Baseline data including age, sex, body mass index, American Society of Anesthesiologists physical status classification, preoperative comorbidities, previous operation history, TNM stage, surgical approach, operation time, gas passage time, and length of hospital stay were obtained. Univariate and multivariate logistic regression analyses were performed to identify risk factors associated with 30-day readmission.

Results: A total of 229 patients who underwent elective colorectal surgery were enrolled. Twenty-four patients were readmitted 30 days after discharge. The most common readmission diagnoses were wound bleeding or surgical site infection. Multivariate analysis indicated that patients who had preoperative hepatic disease were at the highest risk of readmission (odds ratio [OR], 8.98; 95\% confidence interval [CI], 7.35-10.61). Survival outcomes were significantly better in the nonreadmitted group (OS, $\mathrm{P}=0.00$; $\mathrm{DFS}, \mathrm{P}=0.04)$.

Conclusion: This study identified that preoperative comorbidities including hepatic and pulmonary diseases were associated with higher readmission rates after elective colorectal surgery. Moreover, the most common cause of readmission in patients who underwent elective colorectal surgery was wound bleeding or surgical site infection.

\section{Keywords: Colorectal neoplasms; Readmission; Elective surgery; Outcome}

\section{INTRODUCTION}

Over the past few decades, there have been rapid developments in surgical techniques that have played an important role in treatment of colorectal cancer. The type of surgical approach is important for both treatment and evaluating the postoperative recovery of patients with colorectal cancer. This interest in postoperative

Received: August 21, 2019 - Accepted: November 4, 2019

Correspondence to: Jae Kyun Ju, M.D.

Department of Surgery, Chonnam National University Hospital,

42 Jaebong-ro, Dong-gu, Gwangju 61469, Korea

Tel: +82-62-220-6456, Fax: +82-62-227-1635, E-mail: jkju@chonnam.ac.kr ORCID: https://orcid.org/0000-0003-1605-3310

\section{(c) 2020 The Korean Society of Coloproctology}

This is an open-access article distributed under the terms of the Creative Commons Attribution NonCommercial License (https://creativecommons.org/licenses/by-nc/4.0) which permits unrestricted non-

commercial use, distribution, and reproduction in any medium, provided the original work is properly cited. recovery has led to developments in strategies such as minimally invasive approaches, optimal pain control, and aggressive postoperative rehabilitation including early oral feeding and early ambulation. On the basis of these strategies, the concept of enhanced recovery after surgery (ERAS) was introduced [1]. Several studies have shown that ERAS reduces the length of hospitalization and complications after elective colorectal surgery $[2,3]$. However, there is a concern that early discharge might affect other proposed quality measures, including readmission rates. Readmission worsens the physical and psychological burden on patients, wastes social health resources, and increases costs [4]. The readmission rate in patients who underwent elective colorectal surgery ranged between $6 \%$ and $20 \%$ [4-6]. A recent study suggested that readmission rates in patients who underwent elective colorectal surgery over the past 20 years have increased [7]. In addition, length of stay (LOS), comorbidities, and postoperative complications were reported to be closely associated with readmission. Other studies 
suggested that the $\mathrm{C}$-reactive protein (CRP)/albumin ratio is a prognostic factor associated with readmission or short-term survival $[8,9]$.

This study aimed to evaluate the readmission rate in 30 days after elective colorectal surgery and the risk factors that affect the 30-day readmission rate. We specifically focused on the impact of comorbidities to determine factors associated with a higher risk of readmission after elective colorectal surgery. In addition, we aimed to identify causes for readmission, disease-free survival (DFS), and overall survival (OS) in a single institution.

\section{METHODS}

\section{Study population and data source}

This study was conducted in compliance with the principles of the Declaration of Helsinki. The study protocol was reviewed and approved by the Institutional Review Board (IRB) of Chonnam National University Hospital (CNUH-2019-221). Informed consent was waived by IRB.

In this study, the data of 292 patients who underwent elective colorectal surgery for colorectal cancer at Chonnam National University Hospital, South Korea, from December 2010 to December 2015, were retrospectively reviewed. All elective colorectal surgeries were performed by colorectal surgeons with 8-17 years of experience. Patients who (1) underwent right hemicolectomy, left hemicolectomy, anterior resection, lower anterior resection, and Hartmann operation, (2) were 18 years and older, and (3) underwent preoperative examination including colonoscopy with biopsy, chest radiography, electrocardiography, basic blood tests, pulmonary function tests, and abdominal and chest computed tomography scans were included. Magnetic resonance imaging scan was performed as needed. Patients with other malignant diseases or who underwent emergency surgery were excluded from the study.

The baseline characteristics of patients, including age, sex, body mass index (BMI), preoperative American Society of Anesthesiologists (ASA) physical status classification, preoperative comorbidities (such as hypertension, diabetes mellitus, cardiovascular diseases, pulmonary diseases, hepatic diseases, renal diseases, and smoking), previous operation history, clinical TNM stage, type of surgical approach, operation time, gas passage time after the operation, and LOS were obtained. The CRP test was performed on postoperative day 3 , postoperative day 5 , and on the day of discharge. Qualitative data were presented as numbers or percentages, while quantitative data were presented as median or standard deviation.

The primary endpoint of the study was to evaluate the readmission rate in the 30 days following elective colorectal surgery and risk factors that affect 30-day readmission rate.

The secondary endpoints of the study were determination of causes for readmission, DFS, and OS in a single institution. DFS was defined as time from the elective colorectal surgery to either first recurrence or death from any cause. OS was defined as time from the elective colorectal surgery to the date of death from any cause or last follow-up.

\section{Statistical analysis}

Differences between the readmitted group and the nonreadmitted group were determined using Welch t-test for continuous variables and chi-square test or Fisher exact test for categorical variables. Logistic regression analyses were performed to identify independent factors for readmission. Values of $\mathrm{P}<0.05$ were considered significant. Variables with a significance level of $\mathrm{P}<0.10$ based on the univariate analysis and those related to readmission were included in multivariate analysis. In addition, the optimal model was searched using stepwise selection for all variables. Stepwise selection is a step-by-step method from a null model without independent variables to a full model including all variables. Variables with the most effect are included in the model, and variables with effects below the reference point are repeated. It is important to determine the optimal combination of variables. DFS and OS were calculated using the Kaplan-Meier method, and survival curves were compared using the log-rank test. The odds ratios (ORs) and 95\% confidence intervals (CIs) were reported. All statistical analyses were performed using IBM SPSS Statistics ver. 23.0 (IBM Co., Armonk, NY, USA).

\section{RESULTS}

\section{Patient demographics}

The medical data of 292 patients who underwent elective colorectal surgery from December 2010 to December 2015 were retrospectively reviewed. Patients who had no data $(n=21)$ or had not visited the hospital after surgery $(n=35)$ were excluded from this

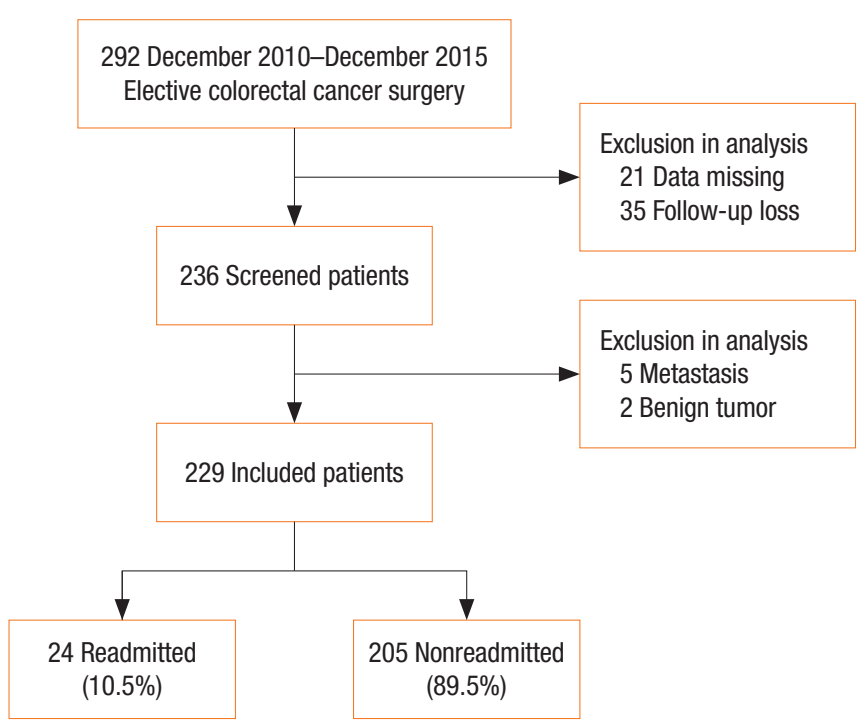

Fig. 1. Patient flow chart. 
study. Patients who were diagnosed with metastatic malignant diseases $(n=5)$ or benign tumors $(n=2)$ were also excluded. A total of 229 patients were finally enrolled in this study (Fig. 1).

The demographics and baseline characteristics of patients are shown in Table 1 . The 30-day readmission rate was $10.5 \%(\mathrm{n}=$ 24). Mean patient age was $64.76 \pm 12.03$ years, and most patients were men $(\mathrm{n}=146,63.8 \%)$. BMI $\left(\mathrm{kg} / \mathrm{m}^{2}\right)$ values were significantly lower in the readmitted group $(\mathrm{P}=0.035)$. There were no significant differences in preoperative comorbidities between the readmitted group and nonreadmitted group. However, higher TNM stage was associated with higher readmission rate $(\mathrm{P}=0.012)$. Most malignant tumors developed in the colon $(n=140,61.1 \%)$.

Table 1. Baseline characteristics of patients

\begin{tabular}{|c|c|c|c|}
\hline Characteristic & $\begin{array}{l}\text { Readmitted } \\
\quad(n=24)\end{array}$ & $\begin{array}{l}\text { Nonreadmitted } \\
\quad(n=205)\end{array}$ & P-value \\
\hline Age (yr) & & & 0.256 \\
\hline$<60$ & $6(25.0)$ & $71(34.6)$ & \\
\hline $60-70$ & $5(20.8)$ & $48(23.4)$ & \\
\hline$>70$ & $13(54.2)$ & $86(42.0)$ & \\
\hline Sex & & & 0.591 \\
\hline Male & $17(70.8)$ & $129(62.9)$ & \\
\hline Female & 7 (29.2) & $76(37.1)$ & \\
\hline Body mass index $\left(\mathrm{kg} / \mathrm{m}^{2}\right)$ & $22.31 \pm 2.56$ & $23.55 \pm 3.10$ & 0.035 \\
\hline ASA PS classification & & & 0.082 \\
\hline I & $2(8.3)$ & $46(22.4)$ & \\
\hline$\|$ & $20(83.3)$ & $150(73.2)$ & \\
\hline III & $2(8.3)$ & $9(4.4)$ & \\
\hline \multicolumn{4}{|l|}{ Preoperative comorbidity } \\
\hline Hypertension & $9(37.5)$ & $69(33.7)$ & 0.882 \\
\hline Diabetes mellitus & $4(16.7)$ & $41(20.0)$ & 0.907 \\
\hline Cardiovascular & $6(25.0)$ & $23(11.2)$ & 0.110 \\
\hline Pulmonary & $5(20.8)$ & $15(7.3)$ & 0.066 \\
\hline Hepatic & $3(12.5)$ & $6(2.9)$ & 0.084 \\
\hline Renal & $3(12.5)$ & $5(2.4)$ & 0.051 \\
\hline Smoking & $4(16.7)$ & $18(8.8)$ & 0.382 \\
\hline Previous operation history & 7 (29.2) & 35 (17.1) & 0.242 \\
\hline Tumor location & & & 0.939 \\
\hline Colon & $14(58.3)$ & $126(61.5)$ & \\
\hline Rectum & $10(41.7)$ & 79 (38.5) & \\
\hline TNM classification & & & 0.012 \\
\hline Stage I & $5(20.8)$ & $88(42.9)$ & \\
\hline Stage \|I & 8 (33.3) & $56(27.3)$ & \\
\hline Stage III & $9(37.5)$ & $61(29.8)$ & \\
\hline Stage IV & $2(8.3)$ & $0(0)$ & \\
\hline
\end{tabular}

Values are presented as number (\%) or mean \pm standard deviation. ASA PS, American Society of Anesthesiologists physical status.

\section{Surgical outcomes}

Table 2 shows the outcomes of surgery. Mean operation time was $146.25 \pm 40.92$ minutes in the readmitted group and $123.07 \pm$ 58.55 minutes in the nonreadmitted group. There was a significant difference in mean operation time between the 2 groups $(\mathrm{P}=$ 0.018). Mean LOS was $10.37 \pm 8.40$ days in the readmitted group and $9.24 \pm 4.98$ days in the nonreadmitted group. There was no significant difference in hospital stay length between the 2 groups $(\mathrm{P}=0.335)$. Other surgical outcomes, including type of surgical approach, gas passage time, and postoperative CRP, albumin, and creatinine levels did not differ significantly between the 2 groups.

\section{Readmission diagnoses}

Readmission diagnoses are shown in Table 3. A total of 24 patients were readmitted within 30 days after discharge. The most common readmission diagnoses were wound bleeding and surgical site infection $(n=10,41.7 \%)$, followed by ileus or intestinal

Table 2. Surgery outcomes

\begin{tabular}{lccc}
\hline Variable & $\begin{array}{c}\text { Readmitted } \\
(\mathrm{n}=24)\end{array}$ & $\begin{array}{c}\text { Nonreadmitted } \\
(\mathrm{n}=205)\end{array}$ & P-value \\
\hline Surgical approach & & & 1.000 \\
$\quad$ Open & $22(91.7)$ & $190(92.7)$ & \\
$\quad$ Laparoscopic & $2(8.3)$ & $15(7.3)$ & \\
Operation time (min) & $146.25 \pm 40.92$ & $123.07 \pm 58.55$ & 0.018 \\
Gas passage time (day) & $2.67 \pm 1.52$ & $3.04 \pm 1.45$ & 0.264 \\
Length of stay (day) & $10.38 \pm 8.40$ & $9.24 \pm 4.98$ & 0.335 \\
CRP on 3 days (mg/dL) & $9.51 \pm 5.40$ & $8.55 \pm 5.00$ & 0.414 \\
CRP on 7 days (mg/dL) & $4.16 \pm 2.97$ & $3.56 \pm 3.74$ & 0.366 \\
CRP on discharge (mg/dL) & $3.40 \pm 2.78$ & $2.83 \pm 3.01$ & 0.346 \\
Albumin (g/dL) & $3.39 \pm 0.46$ & $3.50 \pm 0.39$ & 0.249 \\
CRP(D/C)/albumin ratio & $1.06 \pm 0.91$ & $0.84 \pm 0.94$ & 0.273 \\
Creatinine (mg/dL) & $0.72 \pm 0.22$ & $0.73 \pm 0.52$ & 0.890 \\
\hline
\end{tabular}

Values are presented as number (\%) or mean \pm standard deviation.

CRP, C-reactive protein; D/C, discharge.

Table 3. Readmission diagnoses of 24 patients

\begin{tabular}{lcccc}
\hline Readmission diagnosis & $\begin{array}{c}\text { Colon } \\
(\mathrm{n}=14)\end{array}$ & $\begin{array}{c}\text { Rectum } \\
(\mathrm{n}=10)\end{array}$ & $\begin{array}{c}\text { Total, } \\
\mathrm{n}(\%)\end{array}$ & P-value \\
\hline $\begin{array}{l}\text { Wound bleeding and surgical } \\
\text { infection }\end{array}$ & 7 & 3 & $10(41.7)$ & 0.421 \\
$\begin{array}{l}\text { Ileus and intestinal obstruction } \\
\text { Diarrhea }\end{array}$ & 2 & 3 & $5(20.8)$ & 0.615 \\
Hepatobiliary disease & 2 & 1 & $3(12.5)$ & 1.000 \\
Metastasis & 2 & 0 & $2(8.3)$ & 0.493 \\
Acute kidney injury & 1 & 1 & $2(8.3)$ & 1.000 \\
Other & 0 & 1 & $1(4.2)$ & 0.417 \\
\hline
\end{tabular}


obstruction $(n=5,20.8 \%)$, watery diarrhea $(n=3,12.5 \%)$, and hepatobiliary disease $(n=2,8.3 \%)$. There were 14 patients who underwent colon cancer surgery and 10 patients who underwent rectal cancer surgery in the readmission group. There were no significant differences in readmission diagnoses between the colon cancer group and rectal cancer group.

\section{Risk factors of readmission}

Table 4 shows the results of univariate and multivariate logistic regression analysis on risk factors for readmission. In the multivariate logistic regression analysis, preoperative pulmonary disease, hepatic disease, TNM stage, and operation time were selected by stepwise selection. The results of multivariate analysis indicate

Table 4. Univariate and multivariate logistic regression analysis of risk factors for readmission

\begin{tabular}{lccccc}
\hline \multirow{2}{*}{ Variables } & $\begin{array}{c}\text { Univariate } \\
\text { analysis }\end{array}$ & & \multicolumn{3}{c}{ Multivariate analysis } \\
\cline { 2 - 3 } \cline { 5 - 6 } \cline { 5 - 6 } & P-value & & P-value & OR & $95 \% \mathrm{Cl}$ \\
\hline Body mass index & 0.035 & & NS & & \\
ASA PS classification & 0.058 & & NS & & \\
$\begin{array}{l}\text { Preoperative comorbidity } \\
\quad\end{array}$ & & & & \\
$\quad$ Pulmonary & 0.043 & & 0.013 & 4.90 & $3.65-6.14$ \\
$\quad$ Hepatic & 0.056 & & 0.008 & 8.98 & $7.35-10.61$ \\
$\quad$ Renal & 0.040 & & NS & & \\
TNM classification & 0.025 & & 0.005 & 2.27 & $1.70-2.85$ \\
Operation time (min) & 0.018 & & 0.048 & 1.01 & $1.00-1.01$ \\
\hline
\end{tabular}

ASA PS, American Society of Anesthesiologists physical status; NS, nonspecific; $\mathrm{OR}$, odds ratio; $\mathrm{Cl}$, confidence interval.

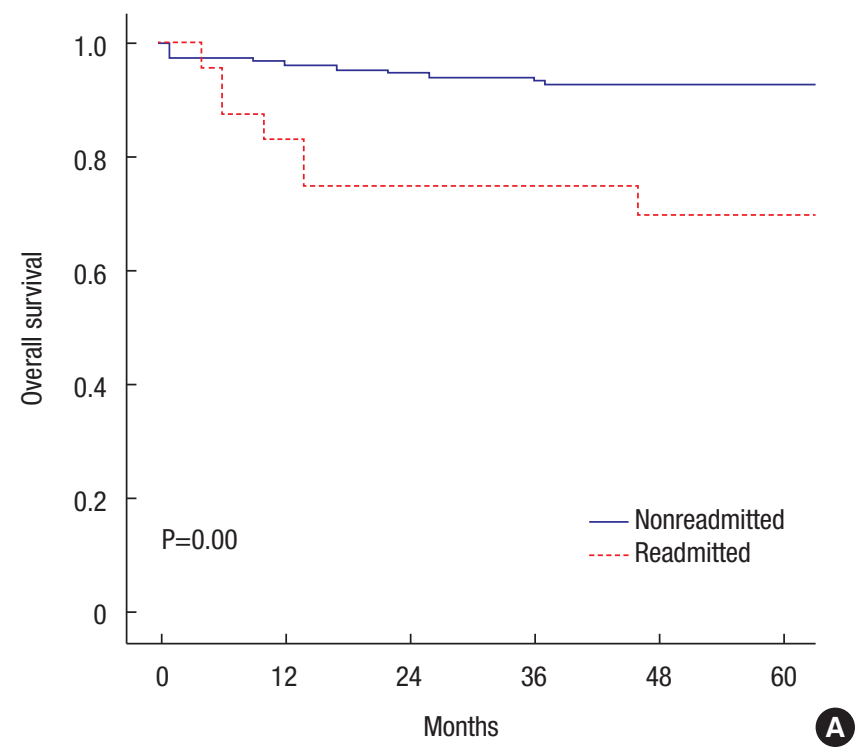

that patients with preoperative hepatic disease were at higher risk of readmission (OR, 8.98; 95\% CI, 7.35-10.61). In addition, pulmonary disease (OR, 4.90; 95\% CI, 3.65-6.14) and TNM stage (OR, 2.27; 95\% CI, 1.70-2.85) were significant predictors of readmission.

\section{Survival outcomes}

The OS and DFS results are represented graphically in Fig. 2. The median follow-up period was 57.8 months. During the follow-up period, 25 patients $(10.92 \%)$ had recurrence $(n=10$, liver metastasis; $\mathrm{n}=5$, lung metastasis; $\mathrm{n}=3$, lymph node metastasis; $\mathrm{n}=1$, mesenteric metastasis; $n=6$, mesenteric metastasis in other sites), whereas 5 patients $(2.18 \%)$ died from other diseases. In the nonreadmitted group, 5-year OS rate was $93.1 \%$ and 5-year DFS rate was $85.7 \%$. In the readmitted group, 5-year OS rate was $70.0 \%$ and 5-year DFS rate was 70.0\%. Kaplan-Meier survival analysis showed a significant difference between the readmitted group and nonreadmitted group (OS, $\mathrm{P}=0.00 ; \mathrm{DFS}, \mathrm{P}=0.04$ ).

\section{DISCUSSION}

Technologic advances in colorectal surgery have increased interest in evaluating the postoperative recovery rate of patients with colon cancer. Unplanned readmission and LOS are widely used as quality indicators of colorectal surgery [10]. In addition, the ERAS program has played an important role in preoperative management of patients undergoing colorectal surgery. The implementation of this program has led to reduced LOS and postoperative complications, early recovery of organ function after surgery, and early return to daily life [11].

This study aimed to assess the readmission rate 30 days after

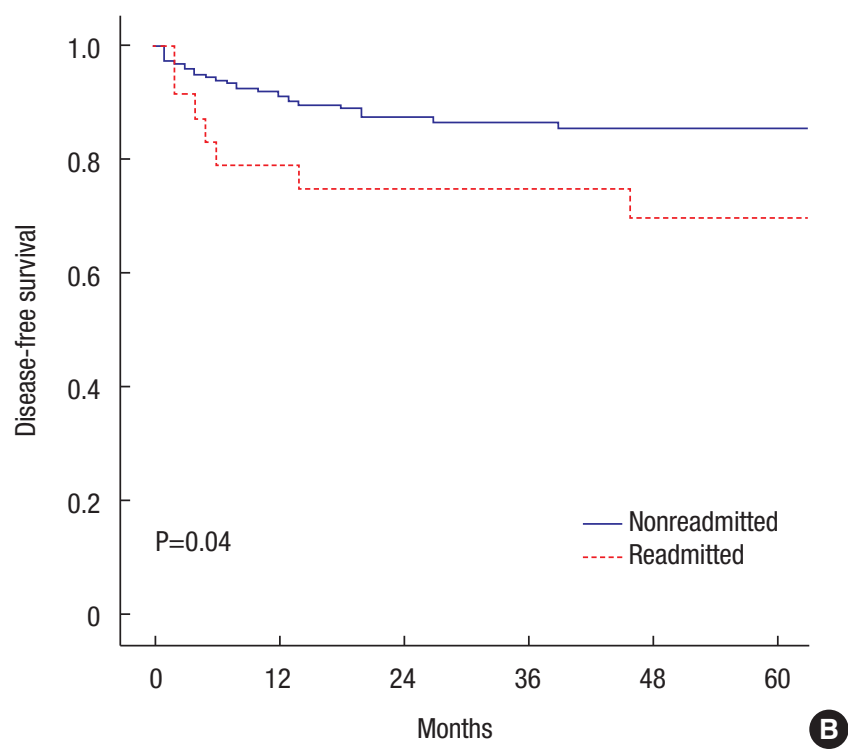

Fig. 2. Kaplan-Meier analyses of overall survival (A) and disease-free survival (B) in patients undergoing elective colorectal surgery. 
elective colorectal surgery and predictive factors related to 30-day readmission. This study showed a 30-day readmission rate of $10.5 \%$, similar to the $9 \%-11 \%$ readmission rates reported in previous studies $[7,12,13]$.

The present study identified 4 statistically significant risk factors for readmission: hepatic disease, pulmonary disease, TNM stage, and operation time. According to our study, patients with preoperative hepatic disease were approximately 9 times more likely to be readmitted (OR, 8.98; 95\% CI, 7.35-10.61). In addition, preoperative pulmonary disease was significantly associated with readmission (OR, 4.90; 95\% CI, 3.65-6.14). Yang et al. [14] reported that patients with preoperative complications were 2.91 times more likely to be readmitted than those without preoperative complications (OR, 2.91; 95\% CI, 1.32-6.99).

However, the factors associated with readmission rate in this study were inconsistent with those reported in previous studies. In our univariate analysis, LOS was not a significant predictor of readmission $(\mathrm{P}=0.335)$. In a few recent studies, LOS was significantly associated with readmission $[7,12]$.

$\mathrm{CRP}(\mathrm{D} / \mathrm{C})$ /albumin ratio was not a statistically significant risk factor for readmission $(P=0.273)$. Oh et al. [15] reported that $\mathrm{CRP} /$ albumin ratio was an independent risk factor for mortality in postoperative patients admitted to the intensive care unit. Xu et al. [16] reported that CRP/albumin ratio was useful for predicting the survival of patients with esophageal cancer.

Our study showed causes of unplanned readmission. The most common causes of readmission were wound bleeding or surgical site infections $(\mathrm{n}=10,41.7 \%)$ and ileus or intestinal obstructions $(n=5,20.8 \%)$. Kariv et al. [13] reported that surgical site infection and ileus were associated with higher readmission rate. A metaanalysis by Li et al. [17] showed that the most common reason for readmission was bowel obstruction or ileus, followed by surgical site infection. Wick et al. [4] reported that one-third of the total number of readmissions in patients who underwent colorectal surgery were considered preventable.

The OS and DFS rates of the nonreadmitted group were significantly higher than those of the readmitted group (OS, $\mathrm{P}=0.00$; $\mathrm{DFS}, \mathrm{P}=0.04)$. Also, the 5 -year survival rate of the readmitted group was relatively low (OS, 70.0\%; DFS, 70.0\%). These results are concordant with previous reports by Schneider et al. [7] and Greenblatt et al. [18].

The present study has several potential limitations. First, our study was a retrospective analysis conducted in a single center; thus, selection bias may exist. Second, the sample size was relatively small $(n=229)$, which limits statistical power. Third, many data for statistical analysis were missing and several patients no longer visited the outpatient clinic, possibly because most patients were aged 60 years or older (66.4\%).

Despite these limitations, this study has important implications. This is the first study to investigate the unplanned readmission rate and risk factors that affect 30-day unplanned readmission rate after elective colorectal surgery in our medical center.
Our study showed that the readmission rate can be reduced by focusing on treatment of wound infection, which is the primary cause of readmission in these patients.

This study provides an impetus for performing multicenter prospective cohort studies in the future to overcome these limitations.

In conclusion, preoperative comorbidities including hepatic and pulmonary diseases were risk factors associated with higher readmission rates after elective colorectal surgery. Moreover, the most common cause for readmission in patients who underwent elective colorectal surgery was wound bleeding or surgical site infection.

\section{CONFLICT OF INTEREST}

No potential conflict of interest relevant to this article was reported.

\section{REFERENCES}

1. Wilmore DW, Kehlet H. Management of patients in fast track surgery. BMJ 2001;322:473-6.

2. Varadhan KK, Neal KR, Dejong CH, Fearon KC, Ljungqvist O, Lobo DN. The enhanced recovery after surgery (ERAS) pathway for patients undergoing major elective open colorectal surgery: a meta-analysis of randomized controlled trials. Clin Nutr 2010;29: 434-40.

3. Adamina M, Kehlet H, Tomlinson GA, Senagore AJ, Delaney CP. Enhanced recovery pathways optimize health outcomes and resource utilization: a meta-analysis of randomized controlled trials in colorectal surgery. Surgery 2011;149:830-40.

4. Wick EC, Shore AD, Hirose K, Ibrahim AM, Gearhart SL, Efron J, et al. Readmission rates and cost following colorectal surgery. Dis Colon Rectum 2011;54:1475-9.

5. Pucciarelli S, Zorzi M, Gennaro N, Gagliardi G, Restivo A, Saugo $\mathrm{M}$, et al. In-hospital mortality, 30-day readmission, and length of hospital stay after surgery for primary colorectal cancer: A national population-based study. Eur J Surg Oncol 2017;43:1312-23.

6. Basse L, Thorbøl JE, Løssl K, Kehlet H. Colonic surgery with accelerated rehabilitation or conventional care. Dis Colon Rectum 2004;47:271-7.

7. Schneider EB, Hyder O, Brooke BS, Efron J, Cameron JL, Edil $\mathrm{BH}$, et al. Patient readmission and mortality after colorectal surgery for colon cancer: impact of length of stay relative to other clinical factors. J Am Coll Surg 2012;214:390-8.

8. Ranzani OT, Zampieri FG, Forte DN, Azevedo LC, Park M. C-reactive protein/albumin ratio predicts 90 -day mortality of septic patients. PLoS One 2013;8:e59321.

9. Miyamoto T, Fujitani M, Fukuyama H, Hatanaka S, Koizumi Y, Kawabata A. The C-reactive protein/albumin ratio is useful for predicting short-term survival in cancer and noncancer patients. J Palliat Med 2019;22:532-7. 
10. Hoffman RL, Bartlett EK, Ko C, Mahmoud N, Karakousis GC, Kelz RR. Early discharge and readmission after colorectal resection. J Surg Res 2014;190:579-86.

11. Melnyk M, Casey RG, Black P, Koupparis AJ. Enhanced recovery after surgery (ERAS) protocols: Time to change practice? Can Urol Assoc J 2011;5:342-8.

12. Kiran RP, Delaney CP, Senagore AJ, Steel M, Garafalo T, Fazio VW. Outcomes and prediction of hospital readmission after intestinal surgery. J Am Coll Surg 2004;198:877-83.

13. Kariv Y, Wang W, Senagore AJ, Hammel JP, Fazio VW, Delaney CP. Multivariable analysis of factors associated with hospital readmission after intestinal surgery. Am J Surg 2006;191:364-71.

14. Yang J, Xiao J, Feng J, Li K. One-year unplanned readmission after colorectal cancer surgery in Western China. J Invest Surg 2019;32: 602-6.
15. Oh TK, Ji E, Na HS, Min B, Jeon YT, Do SH, et al. C-reactive protein to albumin ratio predicts 30-day and 1-year mortality in postoperative patients after admission to the intensive care unit. J Clin Med 2018;7:39.

16. Xu XL, Yu HQ, Hu W, Song Q, Mao WM. A novel inflammationbased prognostic score, the C-reactive protein/albumin ratio predicts the prognosis of patients with operable esophageal squamous cell carcinoma. PLoS One 2015;10:e0138657.

17. Li LT, Mills WL, White DL, Li A, Gutierrez AM, Berger DH, et al. Causes and prevalence of unplanned readmissions after colorectal surgery: a systematic review and meta-analysis. J Am Geriatr Soc 2013;61:1175-81.

18. Greenblatt DY, Weber SM, O'Connor ES, LoConte NK, Liou JI, Smith MA. Readmission after colectomy for cancer predicts oneyear mortality. Ann Surg 2010;251:659-69. 